\title{
La combinación del e.Learning con otras estrategias docentes
}

David Riley, Ainoha Otamendi y José Álvarez

Fundación IAVANTE, Consejería de Salud de Andalucía

El e.Learning como método de enseñanza a distancia debe su éxito a la posibilidad de aprovechamiento de múltiples recursos multimedia proporcionando una formación más encauzada a la intuición y facilitando la labor de aprendizaje a los alumnos. Puede analizarse el e.Learning abordando las variantes que ofrece. Básicamente, en función de la frecuencia de implantancion son dos:

- e.Learning

- Blended learning

E-learning: entendido como autoestudio, teletutorización síncronas y asíncronas. El e.Learning, eminentemente desarrollado en EVA (entorno virtual de aprendizaje) esta especialmente indicado para la transmisión de conocimientos. Idealmente estos cursos sirven a los alumnos para asumir conceptos básicos, la estructura de conocimiento que los capacitará para ahondar en la especialización de su currículo personal, aunque la carga lectiva y

Correspondencia:

D. Riley

Subdirector de teleprestaciones.

Fundación IAVANTE.

david.riley@iavante.es teórica puede ser tan amplia como se considere oportuna. En ellos suele haber una carga importante de contenido conceptual.

B-learning: entendido como la suma de autoestudio, teletutorización y sesiones presenciales. Adecuado para el desarrollo de habilidades y entrenamiento. El objetivo básico de este tipo de cursos es aplicar o adquirir habilidades, destrezas, competencias.

En IAVANTE optamos casi presentemente por esta última modalidad. Pasamos ahora a justificar esta afirmación en base a dos razones principales: una de tipo fundacional y otra, que podemos considerar filosófica.

La razón fundacional podemos buscarla en uno de los valores fundamentales de IAVANTE: la innovación y el entrenamiento. Trabajamos con conocimiento, cocimiento que será tratado y estará dirigido a conseguir la mejora continua en la actividad profesional de los alumnos.

Si la innovación y el entrenamiento se toma como divisa, la organización tiene como tarea esencial la constante búsqueda y aplicación de metodologías novedosas que coadyuven a la consecución del objetivo principal: la mejora continua.

El campo de búsqueda no se circunscribe a ámbito determinado, sino que la intención es integrar y usar 
de forma constructiva todos los medios a nuestro alcance. Es decir, no optamos por una metodología en exclusiva, sino que abogamos por la integración. No, por tanto, innovación por innovación, sino uso innovador de las estrategias y recursos didácticos.

La razón filosófica proviene de una forma de entender el momento actual del desarrollo social, marcado por la sociedad de la información; proceso que no está terminado en absoluto, sino que se encuentra en pleno despliegue de cambios y transformaciones de todos los niveles. A continuación exponemos la teoría de la sociedad del profesor Javier Echevarria, donde presenta la metáfora del tercer entorno, que viene a coincidir con la sociedad de la información. En él, se propugna el cambio necesario que aspectos como la forma de enseñar han de afrontar. ¿Cómo? Adoptando estrategias didácticas que integren metodologías que se han demostrado útiles (y cabe decir que irrenunciables, puesto que nuestra intención es entrenar al profesional) con otras nuevas que surgen al paso del desarrollo de las nuevas tecnologías.

Javier Echevarria, matemático y profesor de filosofía, es uno de los pocos pensadores que, en lengua castellana ha abordado asuntos refereridos a las nuevas tecnologías. En libros como Los señores del aire: Telepolis y el tercer entorno, ha esbozado una teoría que intenta analizar, comprender e interpretar los cambios que, ya es un tópico decirlo, surgen en lo que ha venido a llamarse Sociedad de la Información.

Según el profesor Echevarria el espacio social en el que nos movemos en la actualidad puede analizarse en tres entornos principales que coexisten y se solapan en la confirmación de los elementos que sustentan nuestra vida ${ }^{a}$.

El primer entorno, E1 (lo que rodea, lo que está alrededor) es, en primer lugar, el espacio natural, lo cercano; desde la mera noción de cuerpo, hasta la características propias de la naturaleza: cercanía, necesidad de subsistencia; también puede verse como un primer estadio del desarrollo de la humanidad, un espació primario donde lo más importante es el desempeño en la naturaleza en vista al mantenimiento de la vida.

El E2 es el espacio siguiente, el de la vida en sociedad, en la ciudad, donde las características

a. «Las NTIT posibilitan la construcción de un nuevo espacio social, el tercer entorno (E3), cuya estructura es muy distinta a la de los entornos naturales (E1) y urbanos (E2) en donde tradicionalmente se ha desarrollado la vida social, y en concreto la educación». cambian; por ejemplo, el tiempo de una sociedad agraria esta ligada a los cambios climáticos, el devenir de las estaciones; en cambio, en un sociedad mercantil, industrial, el tiempo está marcado por la producción, los turnos y la productividad, la necesidad de acudir en tiempo y hora a un mismo lugar.

El E3 es el espacio que conforman las redes telemáticas y los servicios y acciones que desarrollan en él. Este E3 tiene características propias que lo diferencia de los anteriores:

- La distancia, que aquí pasa a ser irrelevante (en algunos casos) para actuar, por ejemplo, se puede teletrabajar.

- La "topología", en el sentido de espacio, para actuar debemos estar en un recinto; en cambio, en E3 no hace falta confluir en un mismo sitio.

- La información, la materia de la que está "hecho" este E3; se mueve y se transmite información.

- De forma electrónica; la electrónica predomina en él, frente a E2, por ejemplo, donde la mecánica se impone.

La tesis de la superposición de los entornos, señala el profesor Echeverría, es clave para comprender el paisaje que se dibuja en la sociedad de la información, donde la naturaleza, los diseños industriales y los flujos informacionales generan un nuevo espacio social.

Para vivir en cada uno de ellos el aprendizaje es esencial. En E1, se aprende de manera informal, por uno mismo (sensación de peligro, conocer qué alimento es el idóneo...) o por los demás, (los padres enseñarán a sus hijos a andar, por ejemplo).

En E2, el ámbito de la vida en sociedad es necesario adquirir destrezas y conocimientos que permitan desarrollar y habitar la ciudad, las sociedades. Se crean las escuelas, encargada de transmitir el saber, los principios y doctrinas necesarios en un momento y lugar: desde matemáticas hasta agrimensura. Estos contenidos, estas habilidades y destrezas usaran estrategias didácticas incardinadas en las coordenadas predominantes de tiempo espacio y concurrencia.

En E3 el nuevo espacio social, compuesto por tres medioambientes que conviven, por tres entornos (natural, urbano y electrónico) que se superponen, es un espacio comparable a la polis o la physis. En el tercer entorno, advierte, se encuentran propiedades naturales, industriales y tecnológicas que diseñan una complejidad sin precedentes y que requieren estrategias de aprendizaje igualmente complejas, puesto que estamos obligado a adquirir conoci- 
mientos, cambiantes, y destrezas, nuevas (la alfabetización digital es una necesidad nueva)

El desafío que se nos plantea es adaptar y adaptarnos a las estrategias docentes al tiempo de cambio que estamos viviendo; o sea, "construir el sistema educativo del tercer entorno". Puesto que los tres se solapan y conviven, creemos que lo idóneo es optar por la metodología mixta o, en ingles, blended learning; puesto que debemos seguir entrenando habilidades necesarias en E2, por ejemplo, RCP.

El conocimiento se transforma, se digitaliza; la sociedad pasa a ser informacional, pero no se despega, no se desprende de los estadios anteriores.

\section{COMBINACIÓN DE E.LEARNING Y LA METODOLOGÍA PRESENCIAL: UN EJEMPLO DE BLENDED LEARNING}

Desde el Servicio Andaluz de Salud, la consejería de Salud, y la Fundación IAVANTE, surgió la necesidad de crear un producto innovador que acercase el conocimiento experto a los profesionales del SSPA de forma sencilla y pedagógica. Concretamente, el conocimiento relativo a la Gestión por Procesos Asistenciales Integrados, que constituye una estrategia central del Plan de Calidad de la Consejería de Salud. Desde dicha Consejería, se defiende que el nexo entre profesionales y ciudadanos lo constituye el Proceso Asistencial el cual tiene lugar en diferentes escenarios y con distintos actores ${ }^{\mathrm{b}}$.

La metodología aplicada en el plan de formación es mixta, es decir, tiene una fase presencial y otra no presencial. Es lo que en el entorno de e.Learning se denomina actualmente como "Blended Learning".

Fase presencial: Se explica el uso del CD interactivo como material de autoestudio y la orientación del profesional de cara a la fase virtual. En la formación avanzada se utiliza el caso del CD para generar dinámicas de discusión y análisis de casos entre los alumnos que siguen en la siguiente fase, sobre todo en los grupos compuestos de profesionales multidisciplinares e multinivel (atención primaria y especializada).

Fase no presencial (Autoestudio): Enseñanza a distancia en la que los alumnos/as valiéndose de

b. Tomado de la página web de la Consejería de Salud de la Junta de Andalucía (http://www.csalud.junta-andalucia.es/ principal/documentos.asp?pagina=Procesos_asistenciales) material didáctico elaborado por expertos y entregado en formato CD interactivo, aprenden individualmente, con autoestudio, cada uno de los procesos asistenciales. El CD interactivo contiene los procesos asistenciales del mapa 1 definido por la Consejería de Salud cómo los procesos más prioritarios. Los contenidos están adaptados al entorno de visualización (PC) siguiendo parámetros didácticos conducentes a amenizar el recorrido del proceso. Se trata de dar a conocer el protocolo a seguir desde el punto de vista de un caso concreto (hay una línea argumental en cada explicación que integra las acciones a ejecutar por los distintos perfiles de los profesionales sanitarios). El CD por tanto, se visualiza de forma diferente en función del perfil profesional desde el que accedemos, es decir, el recorrido se adapta en función de las tareas a desempeñar en el proceso por parte de alguno de los integrantes. Los contenidos siguen escrupulosamente las guías de los procesos asistenciales publicados por la Consejería de salud tal y como aparecen en la web oficial www.csalud.junta-andalucia.es/. Profesionales médicos especialistas en las respectivas dolencias y en los procesos asistenciales que abordan el tratamiento son los encargados de la adaptación de los contenidos.

Nada más iniciarse el interactivo, el profesional se encuentra con una breve presentación de la filosofía que sostiene la gestión por procesos. Una vez que el profesional accede a la aplicación, tiene la opción de optar entre dos vías de entrada: por per-

Imagen 1. Esquema-diagrama del proceso asistencial personalizado para el perfil
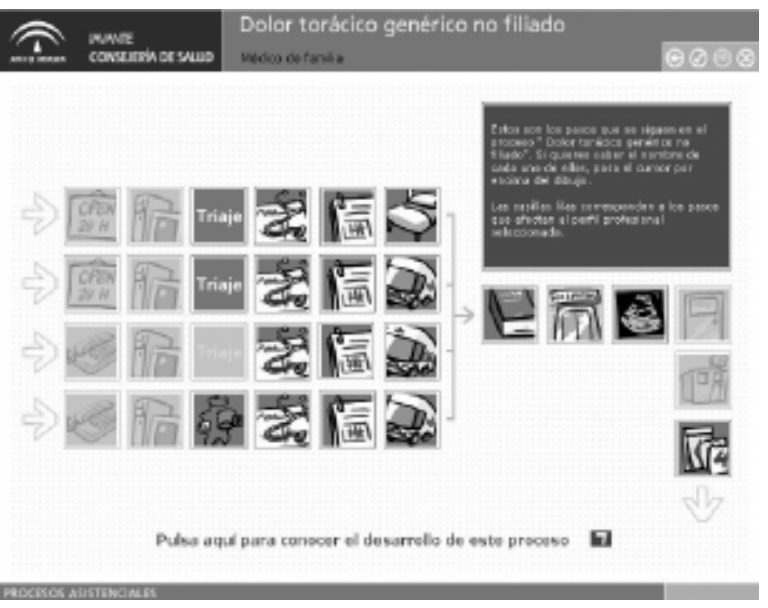
fil profesional -médico, enfermero/a, auxiliar administrativo, etc.- o por proceso asistencial -dolor torácico, diabetes, etc.- de modo que sea el profesional el que decida a qué información quiere acceder y de qué manera.

\section{Imagen 2. Presentación del caso al profesional}

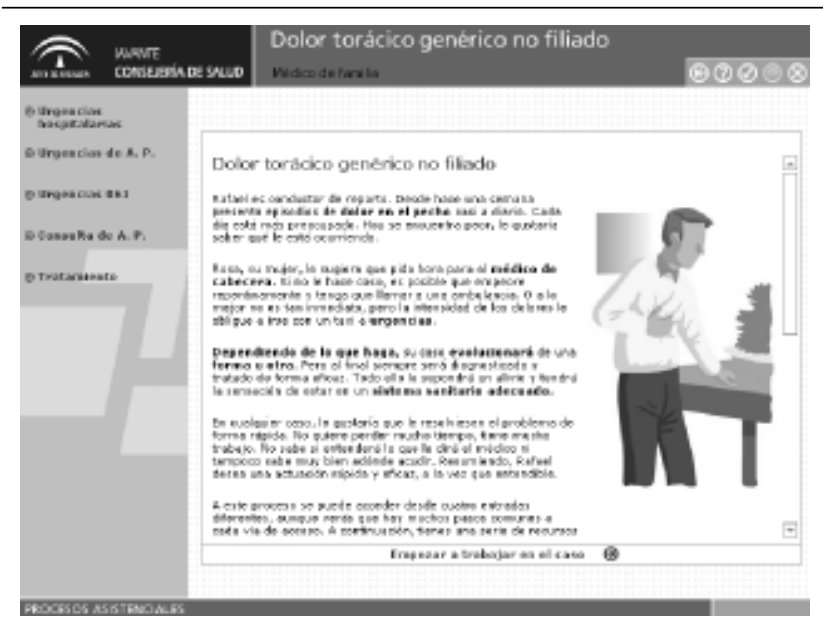

Cuando el profesional ha decidido cómo entrar, el interactivo muestra una pantalla con el esquemadiagrama del proceso. Se colorean los pasos del proceso en los que interviene el perfil seleccionado, y se dejan sombreados los pasos en los que no interviene de manera directa. De todos modos, el profesional siempre puede acceder a todos los contenidos, los colores tan sólo indican aquellos pasos en los que el profesional interviene.

Ya dentro del proceso, el profesional se encuentra, antes de nada, con la presentación de un caso, el de un usuario con nombre, con familiares y amigos que le acompañan y que tienen una serie de expectativas, miedos, etc. La idea es, por tanto, que el profesional viva el proceso como el devenir del ciudadano por el sistema sanitario público. A partir de ahí, el profesional va navegando por el interactivo y trabajando distintas actividades basadas en criterios de calidad, de modo que profundice y aprenda de una manera didáctica los distintos pasos del proceso asistencial, los profesionales que intervienen en ellos y las expectativas del usuario.

Fase no presencial (Teletutorización): Los alumnos de cada acción formativa tienen a su disposición un "micro-entorno" dentro del Entorno Virtual del Proyecto (EVP) donde pueden acceder a herramientas tales como un Foro de Discusión guiado por exper- to, mensajería, enlaces de interés, tablón de anuncio etc., que compone el entorno de Teletutorización del plan de formación. El Entorno Virtual del Proyecto es el sitio web donde residen los recursos de colaboración virtual necesarios para cada uno de los participantes en la formación multidisciplinar en los procesos asistenciales. El acceso al EVP se puede hacer desde www.procesosasistenciales.com, en el enlace "Profesionales SAS" está la entrada a todas las acciones formativas impartidas, divididas por sesión nombrando el lugar y la fecha de la fase presencial. El usuario deberá elegir aquel correspondiente al grupo de trabajo en el que ha participado. El desarrollo del Entorno Virtual del Proyecto (EVP) viene marcado por la índole especial del tipo de formación que se imparte en el proyecto, en cuanto a los contenidos, y de la forma de abordarlos. El EVP es un sitio orientado al debate, ampliación, y puesta en común de las ideas y conocimientos surgidos en las sesiones presenciales, con el objetivo de extender en tiempo y en detalle la formación de las sesiones presenciales.

Tanto la acción formativa básica, como la avanzada están acreditadas por la Agencia de Calidad Sanitaria de Andalucía con 0,74 créditos y 2,48 créditos respectivamente.

\section{COMBINACIÓN DEL E-LEARNING CON LAS NUEVAS METODOLOGÍAS DE SIMULACIÓN}

La simulación es la representación de un proceso o un hecho real mediante otro más simple, que

Imagen 3. Entorno Virtual del Proyecto (www.procesosasistenciales.com)

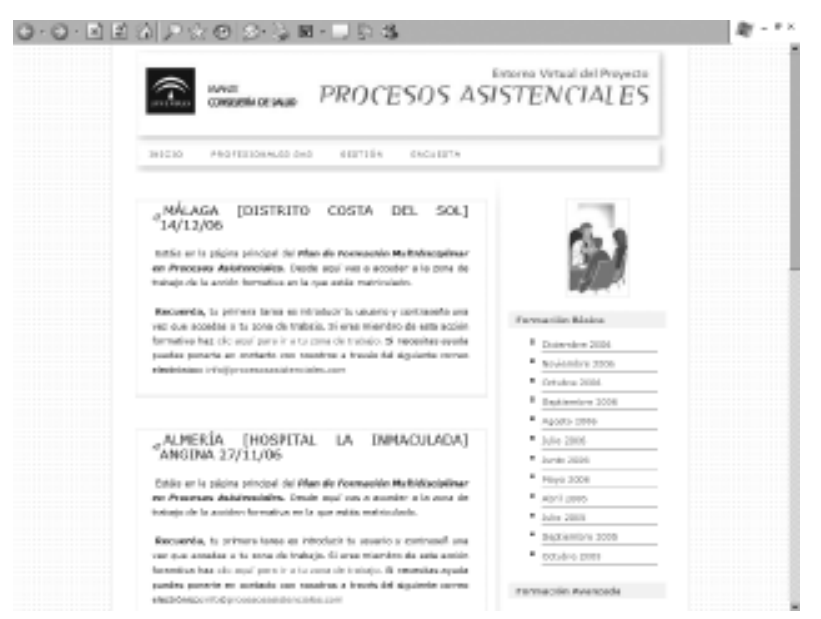


facilita analizar sus características y, por ello, se puede incorporar al proceso de aprendizaje de situaciones complejas en el ámbito del entrenamiento sanitario.

La incorporación de la simulación al entrenamiento clínico del profesional sanitario requiere del desarrollo de nuevas estrategias didácticas que permitan alcanzar los objetivos pedagógicos de cualquier acción formativa que pretenda servirse de estas nuevas metodologías.

Las metodologías de simulación se utilizan fundamentalmente para intentar llevar al discente a un ambiente que reproduzca la realidad y, en este contexto, pueda enfrentarse a situaciones complejas, análogas a las que tenga que resolver en su actividad asistencial.

Por ello, es fundamental que el docente tenga muy claro el diseño, los contenidos, los medios y los objetivos de aprendizaje, lo que sin duda supone un coste elevado y un importante esfuerzo. Esto último es análogo a lo que ocurre con las acciones formativas e-learning, donde se requiere de un diseño y un planteamiento de objetivos pedagógicos claramente especificados antes del comienzo de la acción formativa.

De esto último deducimos, por tanto, que la combinación del e.Learning con metodologías de simulación, requieren de un trabajo excepcional por parte de todos los sujetos implicados en el proceso de enseñanza-aprendizaje, desde la organización, al grupo de docentes, y por último, del alumnado. Esta claro también, que es necesario saber cuándo y por qué vamos a utilizar este tipo de metodologías, pues como ya hemos comentado anteriormente, se suelen utilizar para la adquisición de competencias especí-

Imagen 4. Simulación con Realidad Virtual

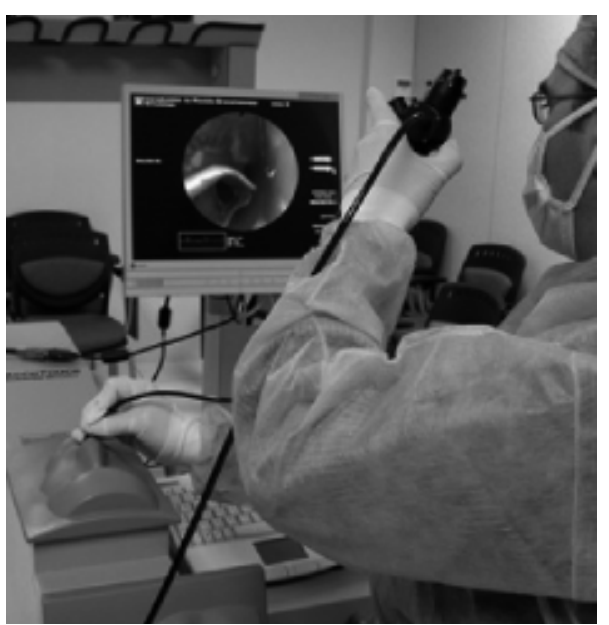

ficas como el manejo de situaciones difíciles, toma de decisiones o habilidades relacionales.

A continuación, mostramos tres tipos de combinaciones entre e.Learning y metodologías de simulación.

\section{Simulación de realidad virtual}

La simulación de realidad virtual se apoya en la utilización de simuladores donde se pueden entrenar habilidades manuales, adquirirse conocimientos teóricos y entrenar la competencia en toma de decisiones. En IAVANTE tenemos varios simuladores de realidad virtual en técnicas endoscópicas, laparoscópicas, y de punción renal con los que entrenamos dichas técnicas a través de distintos casos clínicos.

Una de las combinaciones más fructíferas de este tipo de metodología y el e-learning, es a través de un diseño mixto donde la parte teórica se trabaja en la plataforma de teleformación a través de la presentación de los contenidos -que pueden presentarse en diversos formatos (flash, pdf, Power Point, etc.)junto con el trabajo de dichos contenidos a través del uso de las distintas herramientas e.Learning como los foros y los debates, o los análisis de casos clínicos, bajo la supervisión del docente. Una vez superada esa fase teórica, los alumnos (los profesionales) ya están preparados para comenzar con la parte técnica de la acción formativa, que se trabajarían con los simuladores de realidad virtual en pequeños grupos.

Una importante ventaja de los simuladores de realidad virtual es su capacidad de procesar lo realizado por el alumno, lo que posibilita obtener datos objetivos sobre la ejecución adecuada de las distintas técnicas, para un posterior análisis. Por ello, tras la simulación, se impone una sesión de revisión exhaustiva y debate

\section{Imagen 5. Simulación Robótica}

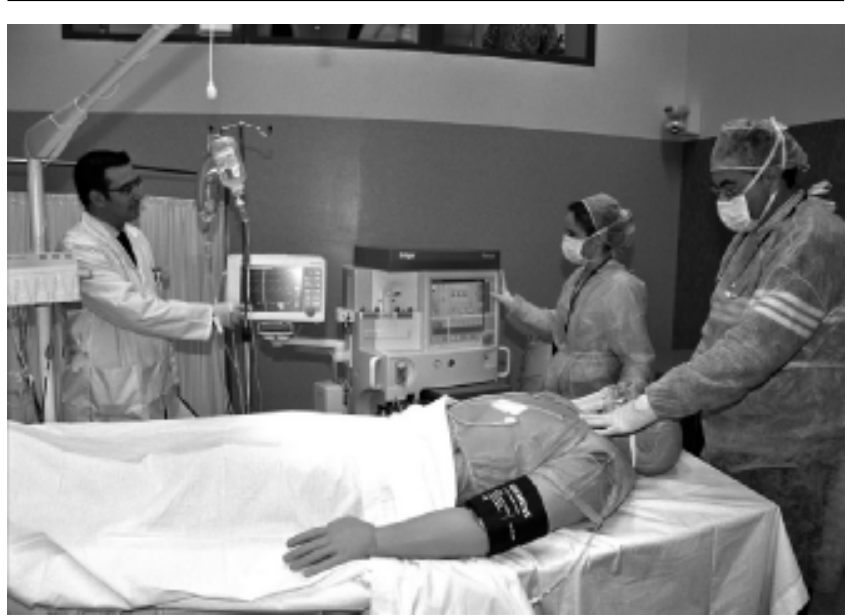


de lo ocurrido, que en u primer momento se realiza en la propia parte presencial, pero que posteriormente puede seguir en la plataforma de teleformación, donde se refuerzan los conceptos aprendidos y las competencias adquiridas en la fase presencial.

\section{Simulación Robótica}

En la simulación robótica las herramientas didácticas son los maniquíes completos -en diferentes grados de complejidad- que simulan pacientes. Los robots más avanzados cuentan con dispositivos electrónicos y mecánicos que permiten la respuesta automática en los signos vitales, dependiendo de la fisiología específica del paciente que simula y las intervenciones clínicas del alumno, siguiendo, para ello, modelos fisiológicos de los sistemas cardiovascular, respiratorio y farmacológico.

La importancia de la Simulación Robótica en el entrenamiento clínico estriba en la posibilidad de trabajar, en situaciones muy próximas a la realidad, en un amplio abanico de procesos asistenciales, tales como, los relacionados a patología respiratoria, cardiaca, trastornos hemodinámicos, trauma grave o tóxicos; además de poder afrontar satisfactoriamente tanto el campo de la anestesiología como el manejo del paciente crítico.

El entrenamiento utilizando la metodología Simulación robótica hace posible la adquisición de competencias de conocimiento teórico y habilidades, principalmente, el manejo de situaciones difíciles, la toma de decisiones, el trabajo en equipo y habilidades técnicas específicas.

$\mathrm{Al}$ igual que en la simulación de realidad virtual, la combinación de esta metodología suele constar de una

\section{Imagen 6. Simulación Escénica}

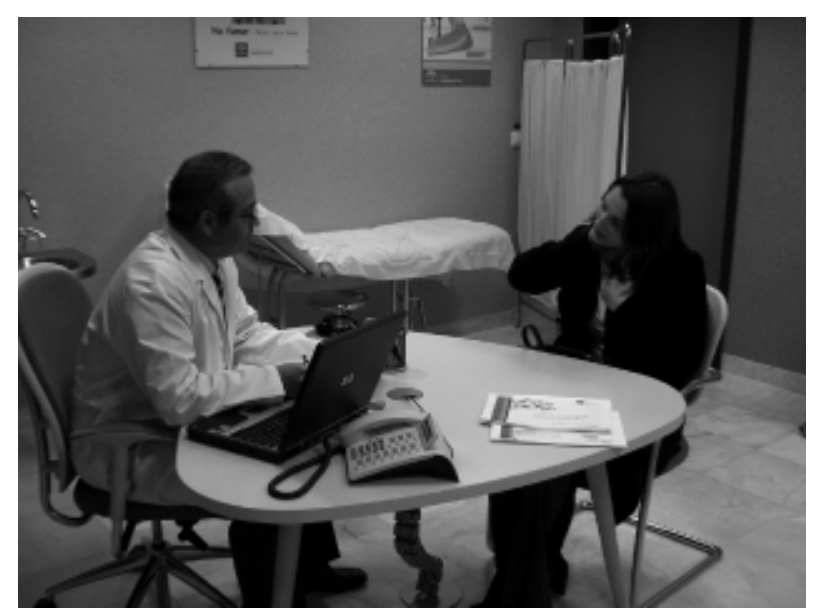

primera fase no presencial donde se tratarán conceptos teóricos, esta vez más relacionados con patologías -no técnicas concretas- y con la toma de decisiones en pacientes críticos. Después se trabajarán los casos en la fase presencial, seguidos de un debate y un videoanálisis. Por último, se puede seguir aprovechando la plataforma de teleformación para reforzar todo lo trabajado en las dos primeras fases; la propia reflexión del discente y la retroalimentación del docente posibilitarán un análisis de las consecuencias de las decisiones tomadas en la simulación.

\section{Simulación escénica}

Con la metodología de simulación escénica se pretende que el profesional-alumno consiga, principalmente, conocimientos teóricos y competencias de habilidades relacionales y el manejo de situaciones difíciles, sobre todo, en el ámbito de la anamnesis, la exploración física, la información al paciente y familiares, y la resolución de conflictos.

En IAVANTE hemos apostado por el entrenamiento de actores que deben, siguiendo un guión preestablecido, interpretar un papel de enfermo con una patología determinada para que simulen situaciones, como las entrevistas clínicas, donde la relación interpersonal tiene una gran repercusión.

En este sentido, y siguiendo la línea de las dos metodologías anteriores, la combinación no presencial + entrenamiento presencial con simulación + cierre no presencial resulta ser la más provechosa.

En resumen, apostamos por la combinación efectiva del e.Learning y las metodologías de simulación innovadoras como una nueva forma de entrenamiento integral de los profesionales sanitarios ${ }^{c}$. De hecho, y aunque aquí las hemos mostrado desglosadas, no tiene por qué entenderse como metodologías excluyentes sino más bien al contrario. Por ejemplo, una acción formativa concreta puede resultar en el entrenamiento de competencias de conocimiento (e.Learning), competencias de habilidad en técnicas concretas (realidad virtual) así como las relacionales (escénica) y las de toma de decisiones (robótica).

c. Para un análisis más detallado del uso de las metodologías de simulación innovadoras, leer a Chaves, J. (2004) "Simulación y entornos de aprendizaje: Entrenamiento competencias clínicas y relacionales". 


\section{CONCLUSIONES}

- Hay pendiente un gran reto de "culturización" hacia el uso de las TIC's en la formación médica.

- La tecnología va por delante de la capacidad de muchas personas para asimilar las nuevas posibilidades y aprovecharlos para la formación.

- e.Learning puro, estaría indicado par un ámbito social del entorno 3, virtual, distal, electrónico. El hecho es que la estructura social no es informacional, sino que esta, la información tiene preeminencia sobre los estadios anteriores el social y natural. Como el proceso esta en desarrollo y nunca desaparecerán la necesidad de adquirir técnica y entrenamiento en procesos, creemos que lo idóneo es la combinación de estrategias docentes con el e-learning.

- Creemos que el e.Learning como concepto, frase y quasi-metodologia, dejara de existir cuando todo el mundo lo tiene asimilado (ya no hablamos de Pizarra-formación, ni retroproyector-formación) o sea que se debe asimilar las nuevas herramientas TIC's como algo normal, necesario y parte integral de la formación.

- El futuro esta en "blended learning" estando el arte en conseguir el blend perfecto, con las cantidades justas de cada elemento-metodología-herramienta.

\section{BIBLIOGRAFÍA}

1. Echeverría, J: Los señores del aire: Telepolis y el tercer entorno. Barcelona: Destino; 1999.

2. Chaves, J: Simulación y entornos de aprendizaje: Entrenamiento competencias clínicas y relacionales [monografía electrónica] 2004. Disponible en URL: http://www.iavante.es/portal3d/html/queofrecemos/monografias_det.asp?codigo $=4$. 